\title{
A Soft Computing Framework for Credit Risk Evaluation
}

\author{
Gafa Lubem, Agaji Iorshase, Esiefarienrhe. B. Micheal
}

\begin{abstract}
Default Credit portfolios have historically been the major cause of financial distress in Nigeria commercial banks because of its inherent risk of possible loan losses (credit risk). It has also contributed to shareholders losing their investment in the commercial banks and inaccessibility of bank loans to the public. Also, evaluations of loan applications by Nigeria Banks are based on a loan officers' subjective assessment. Such assessment is inefficient, lack the ability to learn from customer past financial activities, inconsistent, bias in nature, delay in decision making, unreliable and non-uniform risk assessment which may lead to bankruptcies and defaulted loans. A soft computing framework based on a neurofuzzy model integrated with the customer Bank Verification Number (BVN) to link other Banks was developed. The method used deposit rate, withdrawal rate, average withdrawal, average deposit, loan request amount, collateral value, available cash and average income as inputs which was trained by the neural network to generate four output,that is, collateral, capital, character and capacity. Fuzzy rules were used to evaluate and determine the credit risk level for possible recommendation. The data for this framework was sourced from nine Nigeria Commercial banks. The soft computing framework was implemented using Java enterprise edition programming language. Experimental results showed that the system sufficiently evaluated the credit risk and generated the appropriate risk level with high accuracy for credit recommendation to minimized default loans in the Nigeria Banks.
\end{abstract}

Index Terms - Neurofuzzy, Credit Risk Evaluation, Neural Networks, Fuzzy Set, feed forwarding, learning, frame work, fuzzy inference, nonlinearities, sigmoid function.

\section{INTRODUCTION}

Commercial Banks credits or loans are granted in Nigeria based on unsystematic judgmental using experiences of the bank's credit officers. This approach suffers due to the fact that humans are prone to biasness, inability of the loan officers to examine large and ambiguous volume of customer data, their inability to learn from customer financial activities, long period of evaluating customers credit risk category, personal acquaintances with loan applicants and the failure of the commercial banks to report bad loans to Central Bank of Nigeria (CBN).[1]

This work aim at developing a soft computing frame work

Lubem Gafa, Department of Mathematics/Statistics/ Computer Science, Federal University of Agriculture Makurdi. Makurdi Benue State, Nigeria.

Dr Agaji Iorshase, Department of Mathematics/Statistics/ Computer Science, Federal University of Agriculture Makurdi.Makurdi, Benue State Nigeria.

Dr Esiefarienrhe. B. Micheal, Department of Mathematics /Statistics/Computer Science, Federal University of Agriculture Makurdi,Makurdi, Benue State, Nigeria. using a neurofuzzy model for the evaluation of credit risk for usage by the Nigeria commercial Banks for credit risk rating.

\section{RELATED LITERATURE}

Early credit risk detection is crucial in minimizing the rate of loan defaulters in commercial banks. Many methodologies have been developed in the past in scoring and rating credit risk in commercial banks. In this section we shall examine such methods and their shortcomings.

Capital adequacy, Asset quality, Management ability, Earnings and Liquidity (CAMEL) to ascertain the impact of credit risk on the commercial banks was used by [2]

Credit risk rating is also investigated with three methods including Logistic Regression, Neural Networks and Genetic Algorithms [3] According to their research's result, logistic regression and neural networks are good and similar. Although neural network is slightly better and genetic algorithms take third place.

A knowledge based system prototype to support commercial loan officers in credit risk analysis called LoanAnalysis Support System (LASS) was designed by [4]. This system provided a user interface to deal with variety of data, analytical operations and models in a flexible manner.

A loan evaluation model using support vector machine (SVM) to identify potential applicants for consumer loans was developed by [5]. The experimental results revealed that SVM surpasses neural network models in generalization. The major problem is that the SVM is a complex function and then it is incomprehensible for many humans. Also [6] proposed a comprehension credit scoring using SVM by rule extraction technique. Rules can be extracted from trained SVM that are interpretable by humans and keep as much of the accuracy of the SVM as possible. The results obtained showed that this technique loses only a small percentage in performance compared to SVM and therefore this technique ranks at the top of comprehensible classification techniques.

Theinvestigation of credit risk classification abilities of neural networks, fuzzy logic, genetic algorithms, rule induction software, and rough sets was carried out by [7] and they concluded that the genetic/fuzzy approach compared more favorably with the neurofuzzy and rough set approaches.

The work by [8] presented a comprehensive and systematic approach to developing an optimal architecture of a neural network model for evaluating the credit worthiness of commercial loan applications. The neural network developed using this architecture was capable of correctly classifying $75 \%$ of loan applicants and was superior to neural networks developed using simple heuristics. 


\section{A Soft Computing Framework for Credit Risk Evaluation}

Also [9]presented the results from analyzing three real life credit risk data set using neural network rule extraction techniques. Clarifying the neural network decisions by explanatory rules that captured the learned knowledge embedded in the networks can help the credit risk manager in explaining why a particular applicant is classified as either bad or good. The work also discussed how these rules can be visualized as a decision table in a compact and intuitive graphical format that facilitated easy consultation.

An expert system for the credit administration department at Merrill Lynch to assist setting up of credit limits on margin accounts was developed. Every account with a debit balance over $\$ 100,000$ was delivered daily to the system. The system appraised the account by assessing the risk using a combination of analytical and heuristic reasoning. The system considered the factors like fixed income positions on the basis of quality, marketability and overall portfolio diversity in risk assessment of the account The system warned the credit analyst about problems in the account and gave advice about whether the current debit can be supported and what the credit limits should be[10]

A fuzzy logic model for credit risk detection and evaluation banks using an Excel spreadsheet was operationalized. By using the fuzzy logic model to develop clusters for different statements representing high risk in the detection and rating of credit risk, non-financial data variable was used for the analysis. The overall prediction accuracy for the model was $86.7 \%[11]$.

The adoption ofneurofuzzy model to predict and evaluate the credit risk in Turkish banks was carried out by [12] which sampled fifty five(55) banks. The assessments of these bank performances were based on the group of 24 variable indicators with the level of significance of 5 per cent. This empirical study was conducted using linear regression and non-linear regression on the basis of neurofuzzy logic. The findings from this study indicated that the method using neurofuzzy logic correctly forecast 90.91 per cent whereas using linear regression correctly forecast 81.82 percent.

All the reviewed works failed to use the Customer's Bank Verification Number (BVN) to scan and determine the customers financial past indebtedness status in other banks as part of the evaluation of customer's credit portfolio for appropriate rating and scoring. In Nigeria BVN is used to link a customer to all his bank accounts. Also, the use of Excel Spreadsheet and statistical regression couldn't handle large data influx which usually result in difficulty in learning from ambiguous and unpattern data for appropriate credit risk rating and scoring.

Therefore, in this work a neurofuzzy soft computing framework links customers with their Bank Verification Number (BVN) to determine customer's financial credit status in other Banks before evaluating and scoring of such risk.

\section{METHODOLOGY}

The method used in evaluating the credit risk in this research is a NeuroFuzzy model (NF). The NeuroFuzzy system is adopted to model the uncovering of historical, nonlinear, unpattern, vague, ambiguous and randomly occurring credit risk in Nigeria commercial banks. The system learns by gathering customer historical, pattern, ambiguous financial data using the Neural network pattern of recognition and then activating the explanatory abilities of fuzzy logic with the aid of fuzzy inference rules to study from the financial data in-order to evaluate and make accurate decision to determine the customer's credit worthiness. Also the use of customers BVN to check other bank accounts, payment of utility bills to measure customer's strength.

This study is proposed to use eight standard criteria data of the Nigeria commercial banks. The criteria are deposit rate, withdrawal rate, average withdrawal, average deposit, loan request amount, insurance value, available cash, average and the use of BVN to monitor customer activities in other banks.

\section{A. Design of A Multilayer Soft-Computing} Framework

A multilayer knowledgeable Neurofuzzy model (MLKNF) using Back propagation/feed-forward learning framework is used in this work. The model is used due to its ability to learn from historical data and approximate various nonlinearities with a high degree of accuracy.

The framework is made of a neural network and Fuzzy inference engine fragments. The neural network fragment embraces the raw input layer and the fuzzy inference segment comprises the fuzzization membership layer, if/then rule base layer and the defuzzization/output layer which is being represented by a simple network processing unit called neurons connected by links as shown in figure 1 .

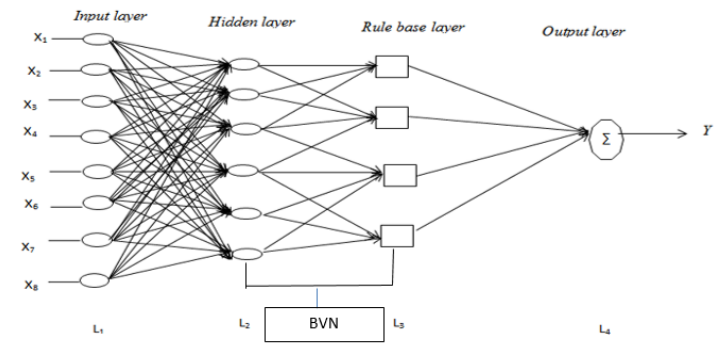

Fig 1.The framework architecture

In fig 1 the input layer has eight neurons, which serve as inputs $\mathrm{x} 1, \mathrm{x} 2 \ldots \mathrm{xn}(\mathrm{n}=8)$ representing the deposit rate, withdrawal rate, average withdrawal, average deposit, loan request amount, collateral value, available balance, average income and the customer BVN use in monitoring customer activities in other banks respectively, plus a bias with various assigned weights $\mathrm{w}_{\mathrm{j}}$ that is been trained with the aid of a neural network function $\varphi$ as represented in Equation 1[13]

$$
\varphi=\left(\sum_{i=1}^{n} w_{i} x_{i}\right)+b_{j}=\left(w^{T} \mathrm{x}\right)(1)
$$

Where $\varphi$ is the activation function for data training, ${ }^{x_{i}}$ are the inputs(Deposit rate, withdrawal rate, average withdrawal, average deposit, loan request amount, collateral value, available cash/balance and average income) fed into the systems, $\boldsymbol{w}_{\boldsymbol{j}}$ is the arbitrary numerical data which is assigned as weights to the inputs in neural network layer(layer 1$). b_{j}$ is additional numerical values of the customer data known as a bias. 
The sigmoid function $f(\varphi)$ in Equation 2 enable/determine the hidden layer2 neurons with nonlinear transfer functions in the network to learn nonlinear relationships between input and output vectors to produce four output (collateral, capital, character and capacity) which is then transferred directly to the surgeno fuzzy inference fragment and become the input variable for the fuzzy inference segments.

$$
f(\varphi)=\frac{1}{1+\exp (-\varphi)}
$$

In equation $2, f(\varphi)$ is the sigmoid function which enables the hidden layer to learn nonlinear relationship between the input-output. $\varphi_{\text {is theoutput from layer1. The output of each }}$ neuron is a function of the weighted sum of the inputs plus a bias. In layer3 from figure 1, the flexible fuzzy inference fragment accepts the output neuron (collateral, capital, character and capacity) as inputs and then uses fuzzy if/then rules in Equation3 to linked each fuzzy set membership to the corresponding fuzzy rule as the consequents with the aid of intersection implemented by the product operator. The defuzzization/output layer uses the consequences of the assimilated by firing the strength of the fuzzy if/then rules which then combine all its output membership into a single fuzzy operation union that ultimately generates the output $y$ using the union function, $f(\Sigma)$. The resulting output from the fuzzy credit risk evaluation is then used to determine the credit risk/credit worthiness of the customer.

The fuzzy rule is proposed because of its capturing If/Then knowledge and facilitating an intuitively-plausible description and its ability to compare and rate linguistic variables.

Notation used for the linguistic variable in the fuzzy rule are: $\mathrm{VP}=$ Very poor, $\mathrm{L}=$ low, $\mathrm{M}=$ Medium, $\mathrm{G}=\mathrm{Good}, \mathrm{H}=$ High, VH= Very high.

Input membership valuesOutput rating Value

Very poor $<=0.30$

Low $>=0.25$ and $<=0.50$

Medium $>=0.50$ and $<0.65$

High $>=0.65$ and $<=0.80$

very High $>=0.80$

Application Statues: Risk Ac Accept: $>=0.50$ and $<=1.00$

Also the notation for the fuzzy inference are defined as: $\left(\alpha_{1}, \beta 1\right)[\Lambda][\mathrm{V}]\left(\alpha_{2}, \beta 2\right)[\Lambda][\mathrm{V}]\left(\alpha_{n}, \beta n\right) \mathrm{VWhere} \mathrm{Y}$ is the antecedent, $\Lambda=$ AND Logic Operator $=$ OR Logic Operator, $\left(\alpha_{1}, \beta 1\right)$ are fuzzy rules, $\alpha$ is a parameter and $\beta$ is a linguistic variable and read as if $\alpha$ is $\beta$. E.g (capital, high) is read capital is high. The Various rules used by the framework are find in Appendix A. In Appendix A. VH, VL, L, H, M, G respectively stands for Very high, Very Low, High, Medium and Good.

In Appendix A, $\alpha_{1}, \alpha_{2} . \ldots \alpha$ arefuzzization input represented as collateral, capital, character and capacity of the train data of the neural network segments; $\beta_{1}, \beta_{2}, \ldots, \beta_{\mathrm{m}}$ are fuzzy sets which range from
$\left\{\begin{array}{l}1,0.5 \leq f(\varphi) \leq 0.9 \\ 0 \text {, otherwise }\end{array}\right.$

$\mathrm{Y}$ is the output obtain from the consequents of the fuzzy rules as the output of the credit portfolio of the customer which could be rejected or accepted.

\section{B. Activity Diagram}

The topology of the soft computing framework model used in this study has eight neurons on the input layer and one neuron on the output layer. This is largely because the network requires eight credit risk criteria attributes to detect, evaluate the risk level involve customer's loan application. The activity diagram of the framework is shown in figure 2.

In figure 2 the framework fetch the inputs (Deposit rate, withdrawal rate, average withdrawal, average deposit, loan request amount, collateral value, available cash/balance and average income). Weights of the inputs are initiated with their various numeric values assign to the weights and additional balance values known as bias. The system then does the training using network activation function $\varphi$. The sigmoid function $f(\varphi)$ which enable the transfer function in the network to learn nonlinear relationships between input and output vectors to produce four output (collateral, capital, character and capacity).which is feed to the fuzzy inference fragment. The BVN of customers is then validate, the if/then fuzzy rule is then used to evaluate the credit risk level. The admin then view the customer loan status if accepted or rejected.

\section{RESULT AND DISCUSSION}

The data used were obtained from Nine Nigeria commercial banks(Access Bank, United Bank For Africa, First Bank, Union Bank, Zenith Bank, Guarantee Trust Bank, Stanbic IBTC and Fidelity Bank) based on the customer's BVN and financial historical transaction data (Deposit rate, withdrawal rate, average withdrawal, average deposit, loan request amount, collateral value, available cash/balance and average income). The data of size 100 was collected without the customer's real personnel information to enable the evaluation and rating of the customer's credit risk level. The collected data is shown in Appendix A. Each row of the data represent Deposit rate, withdrawal rate, average withdrawal, average deposit, loan request amount, collateral value, available cash/balance and average income. The data was normalize to be in the range of zero and one before the network training as shown in Appendix B.

\section{EXPERIMENTAL RESULTS}

The framework was implemented using Java enterprise edition programming language, MySQL Query browser was to provide graphical interface for MySQL server for querying and analyzing data. JDK 1.7.0 was also used as a document generator, archiver during the system implementation. The minimum hardware requirement ensued for the effective implementation of the credit risk evaluation system include; A 64-bit computer system, an Intel Core 3 processor with Radeon HD Graphics $1.40 \mathrm{GHz}, \mathrm{RAM}$ of $4 \mathrm{~GB}$ and Hard Disk of 500GB,Enhanced Keyboard and High-resolution color monitor.

Five (5) experiments were carried out using the framework research. Five different sets of customer's credit risk criteria data obtain with eight inputs corresponding to Deposit rate, withdrawal rate, average withdrawal, average deposit, loan request amount, collateral value, available cash/balance and 
average income were used for the training. A summarized inputs with their corresponding result is shown in Table 1 and table 2. The screenshots below show the sample inputs and the corresponding results of the soft computing framework with a possible recommendation.

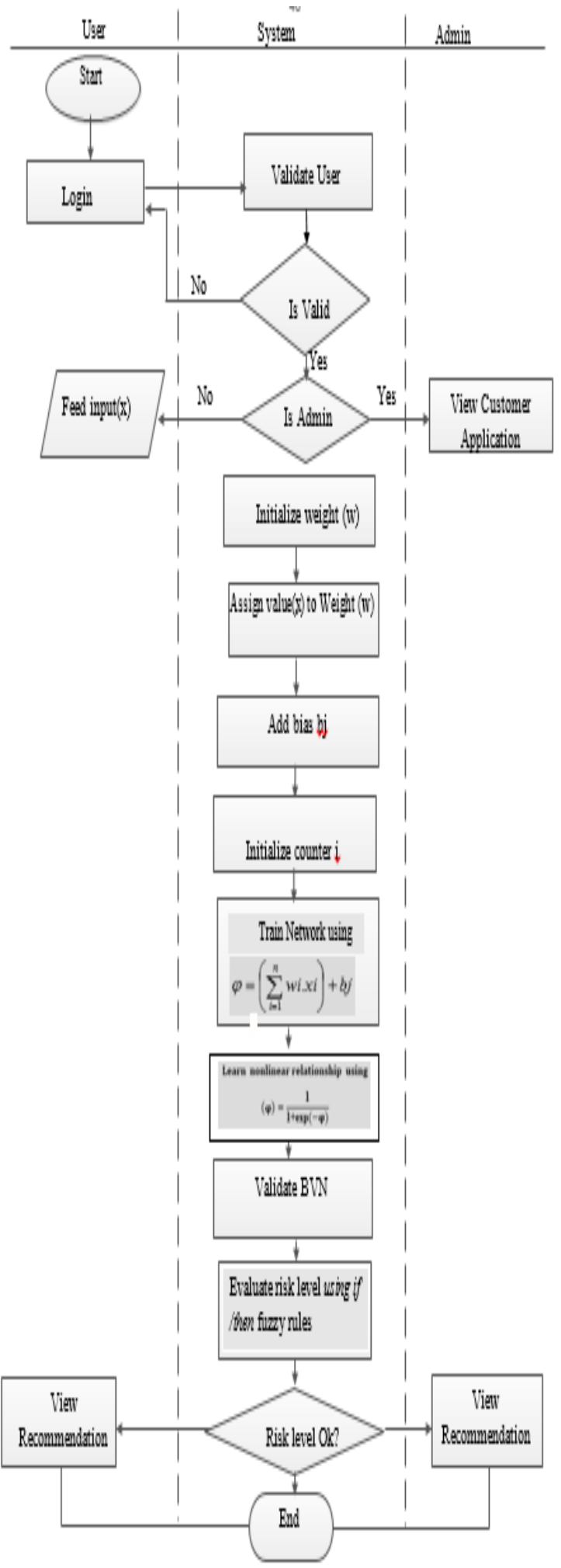

Fig 2.Showing the Activity Diagram of the framework
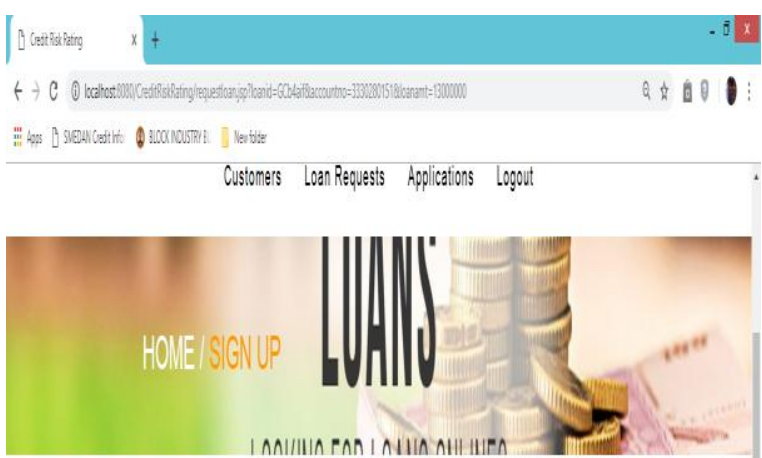

CUSTOUER LOAN REQUEST FOPNI

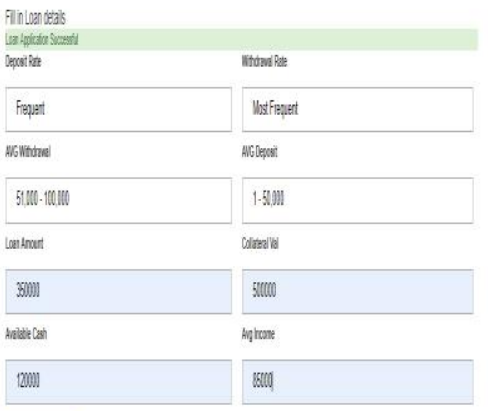

s.brit.

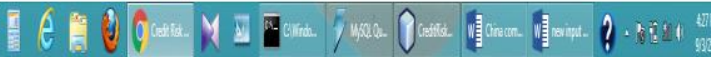

Fig 3. Showing Experiment 1 Sample Inputs.
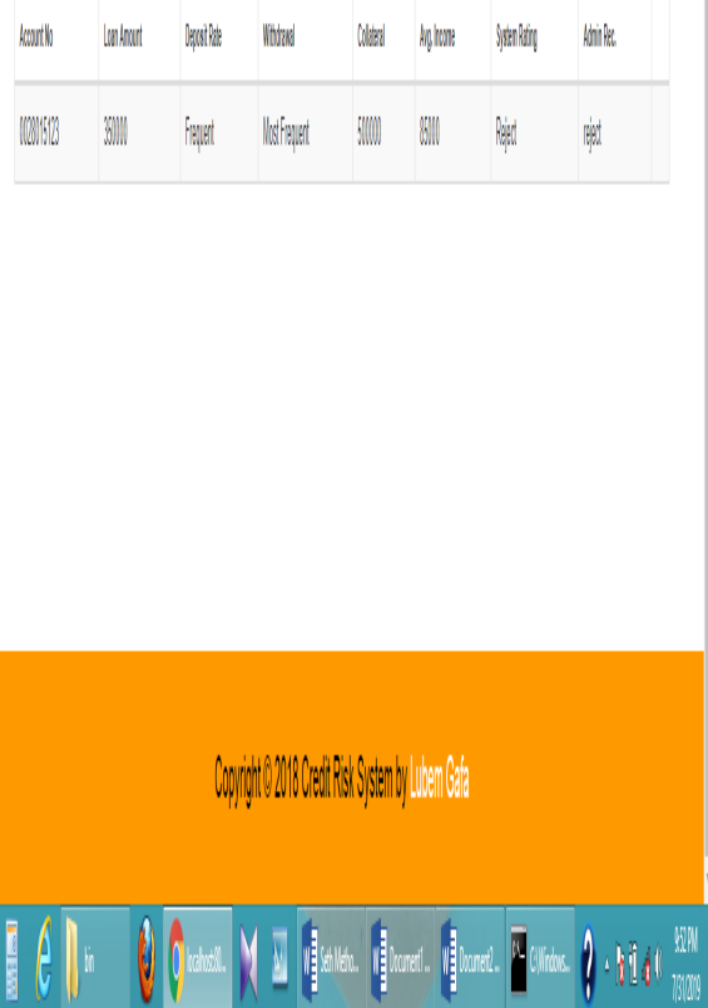

Fig 4: Showing the Sample output for experiment 1 
Table 1:Customers Inputs Values For loan applications

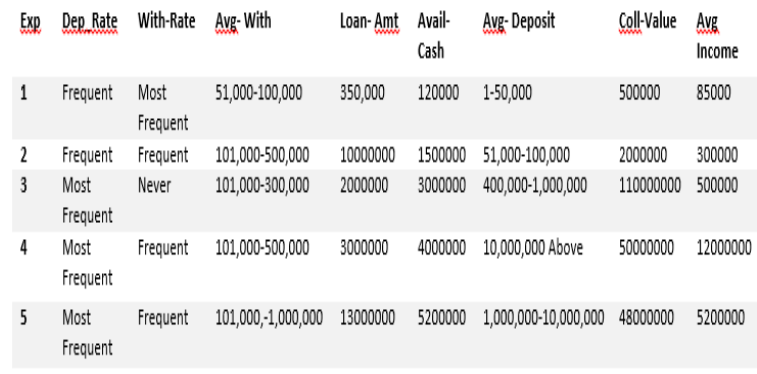

Table 2: Experiments Result.

$\begin{array}{llll}\text { Experiment } & \text { System rating } & \begin{array}{l}\text { Loan Admin } \\ \text { recommendation }\end{array} & \text { Values/Risk Values } \\ 1 & \text { Rejected } & \text { Reject } & 0.20 \\ 2 & \text { Rejected } & \text { Reject } & 0.10 \\ 3 & \text { Accept } & \text { Approve } & 0.80 \\ 4 & \text { Accept } & \text { Approve } & 0.98 \\ 5 & \text { Accept } & \text { Approve } & 1.00\end{array}$

The input values as well as the corresponding outputs of the five experiments conducted are given in table 1 and table 2. where Dep_rate, With_Rate, Avg_With, Loan_ Amt, Avail_Cash , Avg_Deposit, Coll_Value and Avg_Income represents Deposit rate, withdrawal rate, average withdrawal, average deposit, loan request amount, collateral value, available cash/balance and average income respectively. The data in table 1 serve as inputs for the framework while the results in table 2 serve as the result output for the 5 experiments. The use of experiment 1 and experiment 2 show that the customer's loan application were rejected since their credit criteria don't meet the minimum requirements when trained and evaluated. While the use of experiment 3, 4 and 5 indicated that their loan were accepted and approve for disposal since their credit criteria when train/evaluated base on their historical financial record meet the minimum requirement for loan approval.

\section{CONCLUSION}

The proposed model in this framework has proven its effectiveness in determining the credit risk rating of the commercial banks with accuracy. The proposed hybrid neurofuzzy framework base model was able to train from unpattern/ambiguous historical customer information on Deposit rate, withdrawal rate, average withdrawal, average deposit, loan request amount, collateral value, available cash, average income and BVN of customers to determine from other bank the financial transaction of loan applicants, so as to evaluate/rate the risk level in each credit portfolio for possible loan recommendation. The results also show that the proposed neurofuzzy model is scalable, reliable, stable and one of the most significant techniques in Artificial Intelligent that will be used to predict credit risk rating.

\section{RECOMMENDATION}

Experimental results from the work were compared with other loan rating models such as discriminant analysis, Excel Spreadsheet, Z-score, Takagi-sugeno and Mamdani
Evolutionary software (EVoFS).finds show that discriminant analysis, Excel spreadsheet, z-score and EVoFS models lack the threshold to effectively train, evaluate, generate the risk rules for a linguistic explanations of historical nonlinear, unpattern, vague, ambiguous and randomly occurring credit risk data.

The framework is recommended to be used in Nigeria Commercial and Micro finance banks due to its intelligent capabilities to minimize and manage loan defaulters as a result of sniffing credit risk involve in customers ambiguous financial data. Also, in the future, the work can be extended to combine with other artificial intelligence models and statistical tools to explore more intelligent credit risk detection mechanism.

\section{REFERENCES}

[1] Umoh, P. N. (2005), Capital Restructuring of Banks: A Conceptual Framework in CBN (ed) consolidation of Nigeria's Banking Industry: Process of Fourth Annual Monetary Policy Conference, Abuja, FCT.

[2] Shaomei Yang and Junyan Zhao (2009) Study on Commercial Banks Credit RiskBased on AGA and Camel Rating System. Second International Workshop on Knowledge Discovery and Data Mining 978-0-7695-3543-2/09 2009 IEEE DOI 10.1109/WKDD.2009.62

[3] Gouvêa, M. P. \&Gonçalves, E. B. (2007). Credit Risk Analysis Applying Logistic Regression, Neural Networks and Genetic Algorithms Models.Paper presented at the Production and Operations Management Society (POMS), Dallas, Texas, U.S.A.

[4] Duchessi\&Belardo (1987) Lending Analysis Support System (LASS): an application of knowledge based system to support commercial loan analysis margin credit evaluation system, IEEE Transactions systems, man and Cybernetics, 17 (4): 608-616.

[5] Li S, Shiue W and Huamg M (2006). The evaluation of consumer loans using support vector machines. Expert Systems with Applications, 30: 772-782.

[6] Martens, D., Baesens, B,,Gestel, T V. and Vanthienen, J.(2007). Comprehensible credit scoring models using rule extraction from support vector machines, European Journal of Operational Research, 183(3): 1466 - 1476.

[7] Jagielska I. and Jaworski J (1999), An Investigation into the Application of Neural Networks, Fuzzy Logic, Genetic Algorithms, and Rough Sets to Automated Knowledge Acquisition for Classification Problems, Neurocomputing: An International Journal 24(1-3): 37-54.

[8] Glorfeld, L.W., and Hardgrave, B.C.( 1996) An Improved Method for Developing Neural Networks: TheCase of Evaluating Commercial Loan Credit Worthiness, Computer \& Operations Research, 23(10): 933-944

[9] Yang B, Li X L. Ji H and Xu J (2001)An Early Warning System for Loan Risk Assessment Using Artificial Neural Networks. Knowledge-Based Systems 14(5-6): 303-306.

[10] Beshinske.R Stephen R S, Raj N and Margin (1999) CreditEvaluation System, TH0399-6191/0000/0128\$01.00 8 1991 IEEE :128-131.

[11] Lenard M J , Alam P, Booth D and Madey G (2001) Decision-making Capabilities of a Hybrid System Applied to the Auditor's Going-Concern Assessment. Intelligent Systems in Accounting, Finance, and Management: An International Journal 10 (1):1 -24.

[12] Yildiz, B. and Akkoc, S. (2010) Bankruptcy Prediction Using Neuro Fuzzy: An Application in Turkish Banks", International Research Journal of Finance and Economics, Issue(60):114-126

[13] Aggarwal.(2018).Neural Networks and Deep Learning: A Textbook (Springer). 
Appendix A: Fuzzy Rules Used for the Soft Computing Framework

Rule1:IF(collateral,VH) $\Lambda$ (capital,, $\mathrm{VH}) \Lambda$ (capacity, $\mathrm{VH}) \Lambda$ (character,VH)

THEN ( risk,VL). (Accept)

Rule2:IF(collateral,VL) $\Lambda$ (capital,VL) $\Lambda$ (capacityVL) $\Lambda$ (character,VH)

THEN( risk VH). (Reject)

Rule3:IF(collateral,VH) $\Lambda$ (capital,VH) $\Lambda$ (capacity,VH) $\Lambda$ (character,M)

THEN (risk,L). (Accept)

Rule4:IF(collateral,VH) i $\Lambda$ (capital,VH) $\Lambda$ (capacity,VH) $\Lambda$ (character,L)

THEN (risk,G). (Accept)

Rule5:IF(collateral, $\mathrm{VH}) \Lambda$ (capital, $\mathrm{VH}) \Lambda$ (capacity, $\mathrm{VH}) \Lambda$ (character, $\mathrm{VP})$

THEN (credit,G). (Accept)

Rule6: $\quad \operatorname{IF}(\quad$ collateral, $\mathrm{VH}) \quad \Lambda \quad($ capital,, $\mathrm{VH}) \quad \Lambda($ Capacity, $\mathrm{H})$

$\Lambda$ (character,VH)THEN (risk,L). (Accept)

Rule7:IF(collateral,VH) $\Lambda$ (capital,VH) $\Lambda$ (capacity,M) $\Lambda$ (character,VH)

THEN ( risk,L). (Accept)

Rule8:IF(collateral, $\mathrm{VH}) \Lambda($ capital, $\mathrm{VH}) \Lambda$ (capacity,M) $\Lambda$ (character, $\mathrm{VH})$

THEN (risk,G). (Accept)

Rule9: IF(collateral,VH) $\Lambda$ (capital,VH) $\Lambda$ ( Capacity,L $) \Lambda$ (character,VH)

$\Lambda$ THEN (risk,G).(Accept)

Rule10:IF(collateral,VH) $\Lambda$ (capital,VH $\Lambda$ (capacity,VL) $\Lambda$ (character,VH)

$\Lambda$ THEN ( risk,G).(Accept)

Rule11:IF(collateral,VH) $\Lambda$ (capital,H) $\Lambda$ (capacity,VH) $\Lambda$ (character,VH)

THEN (risk,VL). (Accept)

Rule12:IF(collateral, $\mathrm{VH}) \Lambda$ (capital,M) $\Lambda$ (capacity, $\mathrm{VH}) \Lambda$ (character, $\mathrm{VH})$

THEN (risk,G).(Accept)

Rule13:IF(collateral, $\mathrm{VH}) \Lambda$ (capital,L) $\Lambda$ (capacity,, $\mathrm{HH}) \Lambda$ (character, $\mathrm{VH})$

THEN (risk,G).(Accept)

Rule14: IF(collateral,VH) $\Lambda$ (capital,VL) $\Lambda$ (capacity,VH) $\Lambda$ (character,VH)

THEN (risk,H). (Reject)

Rule15:IF(collateral,H) $\Lambda$ (capital,, $\mathrm{VH}) \Lambda$ (capacity, $\mathrm{VH}) \Lambda$ (character,VH)

THEN( risk,L). (Accept)

Rule16: IF(collateral,M) $\Lambda$ (capital,VH) $\Lambda$ (capacity, $\mathrm{VH}) \Lambda$ (character,VH)

THEN (risk,L). (Accept)

$\Lambda$ (capacity, $\mathrm{VH}) \Lambda$ (character,VH) THEN (risk,G) , (Accept)

Rule18:IF(collateral,VP) $\Lambda$ (capital,VH) $\Lambda($ capacity,, $\mathrm{HH}) \Lambda$ (character,VH)

THEN (risk,G). (Accept)

Rule 19:IF(collateral,H) $\Lambda$ (capital,H) $\Lambda$ (capacity,H) $\Lambda$ (character,H) THEN

(risk,Low). (Accept)

Rule20: IF(collateral,H) $\Lambda$ (capital,H) $\Lambda$ (capacity,H) $\Lambda$ (character,M) THEN

(risk,G). (Accept)

Rule 21: $\mathrm{IF}$ (collateral,H) $\Lambda$ (capital,H) $\Lambda$ (capacity,H) $\Lambda$ (character,L) THEN

(risk,G). (Accept)

Rule22: $\mathrm{IF}($ collateral,P) $\Lambda$ (capital,P) $\Lambda$ (capacity,H) $\Lambda$ (character,L) THEN

(risk,H) . (Reject)

Rule23:IF(collateral,H) $\Lambda$ (capital,H) $\Lambda$ (capacity,H) $\Lambda$ (character,, $\mathrm{LL})$

THEN (risk,H). (Reject)

Rule24:IF(collateral,H) $\Lambda$ (capital,H) $\Lambda$ (capacity,M) $\Lambda$ (character,H) THEN

(risk,M).(Accept)

Rule25: $\mathrm{IF}($ collateral,H) $\Lambda$ (capital,H) $\Lambda$ (capacity,L) $\Lambda$ (character,VL)

THEN (risk,H). (Reject)

Rule 26: $\mathrm{IF}($ collateral,H) $\Lambda$ (capital,H) $\Lambda$ (capacity, $\mathrm{VL}) \quad \Lambda$ (character,H)

THEN (risk,H) .(Reject)

Rule27:IF(collateral,H) $\Lambda$ (capital,M) $\Lambda$ (capacity,H) $\Lambda$ (character,H)

THEN (risk,L) .(Accept)

Rule28: IF(collateral,H) $\Lambda$ (capital,L) $\Lambda$ (capacity,H) $\Lambda$ (character,H)THEN

(risk,G) .(Accept)

Rule 29: $\mathrm{IF}($ collateral, $\mathrm{H}) \quad \Lambda$ (capital, $\mathrm{VL}) \quad \Lambda($ capacity, $\mathrm{H}) \quad \Lambda$ (character, $\mathrm{L})$

THEN (risk,H) .(Reject)

Rule 30:IF(collateral,M) $\Lambda$ (capital,H) $\Lambda$ (capacity, $\mathrm{VP}) \quad \Lambda$ (character,P)

THEN (risk,L) .(Accept)

Rule31:IF(collateral,L) $\Lambda$ (capital,H) $\Lambda$ (capacity,H) $\Lambda$ (character,H) THEN

(risk,H). (Reject)

Rule32: $\mathrm{IF}($ collateral,VL) $\Lambda$ (capital,H) $\Lambda$ (capacity,H) $\Lambda$ (character,H)

THEN (risk,H) .(Reject)

Rule33:IF(collateral,M) $\Lambda$ (capital,M) $\Lambda$ (capacity,M) $\Lambda$ (character,M)

THEN (risk,G) .(Accept)

Rule34:IF(collateral,M) $\Lambda$ (capital,M) $\Lambda$ (capacity,M) $\Lambda$ (character,, $\mathrm{H})$

THEN (risk,L) .(Accept)

Rule35:IF(collateral,M) $\Lambda$ (capital,M) $\Lambda$ (capacity,M) $\Lambda$ (character,H)

THEN (risk,L) (Accept)

Rule36: $\mathrm{IF}($ collateral,M) $\Lambda$ (capital,M) $\Lambda$ (capacity,M) $\Lambda$ (character, $\mathrm{VH})$

THEN (risk,L) (Accept)

Rule37:IF(collateral,M) $\Lambda$ (capital,M) $\Lambda$ (capacity,M) $\Lambda$ (character,L)

THEN (risk,G) (Accept)

Rule38: $\mathrm{IF}($ collateral,M) $\Lambda$ (capital,M) $\Lambda$ (capacity,M) $\Lambda$ (character,VL)

THEN (risk,G). (Accept)

Rule 39: $\mathrm{IF}($ collateral,VP) $\Lambda$ (capital,VP) $\Lambda$ (capacity,VP) $\Lambda$ ( character,VP)

THEN (risk,VP) .(Reject) 